\title{
High-power and time-reversed ultrasound based on FEM simulations and experiments
}

\section{Mustonen, Joonas Aleksi}

\section{IEEE}

2019

Mustonen , J A , Tommiska, O M , Rauhala , T , Moilanen , P , Gritsevich , M , Salmi , A \& Haeggström , E 2019 , High-power and time-reversed ultrasound based on FEM simulations and experiments . in 2019 IEEE International Ultrasonics Symposium (IUS) . IEEE, pp. 2458-2460 , IEEE International Ultrasonics Symposium , Glasgow , United Kingdom , 06/10/2019 . https://doi.org/10.1109/ULTSYM.2019.8925992

http://hdl.handle.net/10138/326122

https://doi.org/10.1109/ULTSYM.2019.8925992

acceptedVersion

Downloaded from Helda, University of Helsinki institutional repository.

This is an electronic reprint of the original article.

This reprint may differ from the original in pagination and typographic detail.

Please cite the original version. 


\section{High-power and time-reversed ultrasound based on FEM simulations and experiments}

\author{
Joonas Mustonen \\ Electronics Research Laboratory \\ Physics Department, University of \\ Helsinki \\ Helsinki, Finland \\ joonas.mustonen@helsinki.fi
}

\author{
Oskari Tommiska \\ Electronics Research \\ Laboratory \\ Physics Department, University \\ of Helsinki \\ Helsinki, Finland \\ oskari.tommiska@helsinki.fi
}

\author{
Timo Rauhala \\ Altum Technologies Oy \\ Helsinki, Finland \\ timo.rauhala@altumtechnologies \\ .com
}

Maria Gritsevich

Electronics Research Laboratory

Physics Department, University of Helsinki

Helsinki, Finland

maria.gritsevich@helsinki.fi

\author{
Ari Salmi \\ tronics Research \\ Laboratory \\ Physics Department, University \\ of Helsinki \\ Helsinki, Finland \\ ari.salmi@helsinki.fi
}

\author{
Edward Hæggström \\ Electronics Research \\ Laboratory \\ Physics Department, University \\ of Helsinki \\ Helsinki, Finland \\ edward.haeggstrom@helsinki.fi
}

\author{
Petro Moilanen \\ Electronics Research Laboratory \\ Physics Department, University of \\ Helsinki \\ Helsinki, Finland \\ petro.moilanen@altumtechnologie \\ s.com
}

Abstract-Time-reversal (TR) is method to focus acoustic energy inside complex media. TR focusing to a predefined target point requires either an echo from a target point with acoustic impedance contrast or direct impulse actuation at the target point. Here, we used FEM to simulate the direct impulse actuation and focused ultrasound successfully to different locations in homogenous media with only four narrowband Langevin transducers. Measured intensities indicate that the focal point of the acoustic field was translated and the pressure scan shows that our TR technique works both in simulation and in experimental domain.

Keywords-Time-reversal, focusing, actuation, high-power, FEM, simulations

\section{INTRODUCTION}

Time reversal (TR) is a focusing technique, in which received acoustic waves are transmitted backwards, to coalesque onto the original source of the wave [1]. This is possible due the reciprocity principle, which occurs from the acoustic wave equation containing only even order derivates [1]. Therefore for each spatial and temporal solution $\mathbf{p}(\mathbf{r}, \mathrm{t})$ there is also another solution with negative time $\mathbf{p}(\mathbf{r},-\mathbf{t})$. If one acoustic wave propagates a certain path from $\mathrm{A}$ to $\mathrm{B}$, a wave could propagate from $\mathrm{B}$ to $\mathrm{A}$ by transmitting its received version as timereversed. The TR technique requires no detailed information about the propagation medium. This is utilized in e.g. medical applications, such as lithotripsy[2] and brain stimulation therapy [3].

Usually in a TR experiment received acoustic waves are produced with direct impulse actuation at the target point [4]. Tight spatial focusing requires an array of transducers coupled such that they encase the target point, especially in homogenous media [1]. We present an alternative approach: we simulate the forward propagating waves using FEM and demonstrate that focusing is achieved in a real experimental system using these synthetic signals. This addresses one basic issue with the classical TR approach: the need to get the TR forward signal generated from inside the sample.

\section{MATERIALS AND METHODS}

\section{A. Experimental setup}

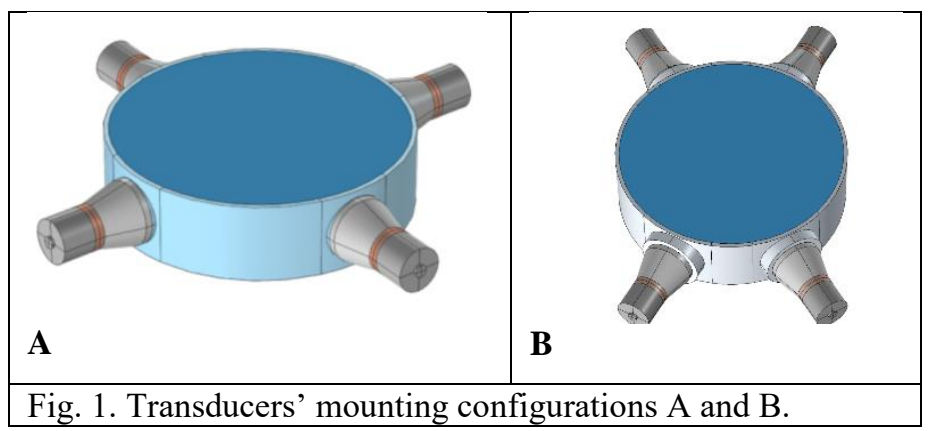

In experiments, four narrowband Langevin transducers (20 $\mathrm{kHz}, 100 \mathrm{~W}$, PZT-8, Beijing Ultrasonics) were coupled symmetrically on a cylindrical Plexiglas container (ø $300 \mathrm{~mm})$. Simulated TR signals were downloaded from computer to sound card (ASUS Xonar U7, bandwidth $193 \mathrm{kHz}$ ) and transmitted to transducers via an amplifier (High-Density 6000 Watt power amplifier) and impedance-matching box. The TR signals featured $V_{p p}=200 \mathrm{~V}$ and the signals were pulsed with $P R F=0.33$ Hz. The setup was controlled with custom-made Python 
software (3.5). We used two mounting configurations A and B, to see if the configuration affects the focusing capability.

\section{B. Simulated impulse actuation}

In simulations, TR signals were created in COMSOL Multiphysics (5.4). The signals were generated by simulating 20 $\mathrm{kHz}$ excitations at the target point and by recording the echoes with four transducer models. The FEM simulation model mimicked the experimental setup; including container's size, materials and transducers' properties. Before transmitting in the experimental domain, the simulated TR signals were amplified.

\section{TABLE 1. LIST OF SETUP PROPERTIES}

\begin{tabular}{|c|c|}
\hline $\begin{array}{c}\text { Container size (radius } \mathrm{x} \\
\text { height) }\end{array}$ & $300 \mathrm{~mm} \times 500 \mathrm{~mm}$ \\
\hline Container material & Plexiglass \\
\hline Transducers & PZT-8, $20 \mathrm{kHz}, 100 \mathrm{~W}$ \\
\hline
\end{tabular}
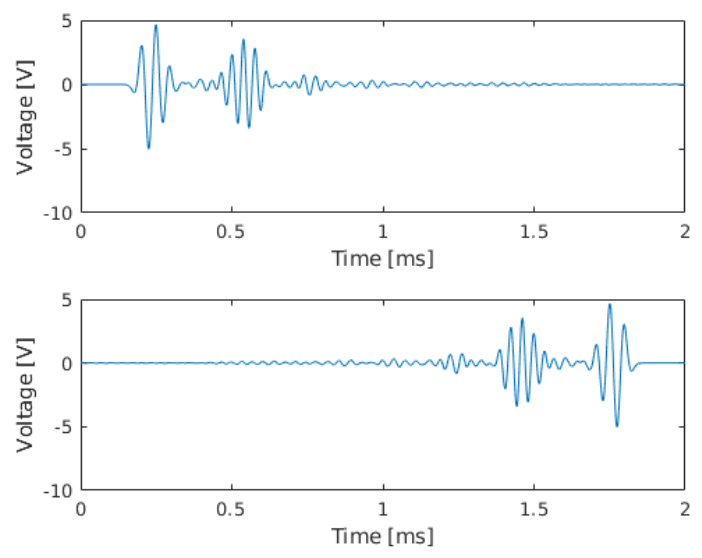

Fig. 2. Example of simulated, TR signal before amplification.

\section{Determining focal points}

There are several ways to determine focal points of focused ultrasound [5]. Here, we compared spatial-peak, temporal-peak intensities, and peak-negative pressures. Spatial-peak, temporalpeak intensity describes the maximum value of acoustic energy in time and space [6].

$$
I_{s p t p}=|\boldsymbol{p}(\boldsymbol{r}, t)|_{\max ^{2}}^{2} / c \rho
$$

where $I_{\text {sptp }}=$ spatial-peak, temporal-peak intensity, $\mathbf{p}(\mathbf{r}, \mathrm{t})=$ space and time dependent pressure, $\mathrm{c}=$ phase speed of sound and $\rho=$ density.

A large peak-negative pressure is necessary in order to produce inertial cavitation [7]. Therefore maximum absolute values of peak-negative pressures were investigated.
With transducers' configuration A, six (6) different locations were investigated as target points and TR signals were produced in the FEM domain. The TR signals were transmitted with the aforementioned experimental setup. Intensities were calculated based on measured pressures at the same six locations with miniature, omnidirectional hydrophone (Brüel \& Kjær type 8103). A high intensity pressure value indicates focal point.

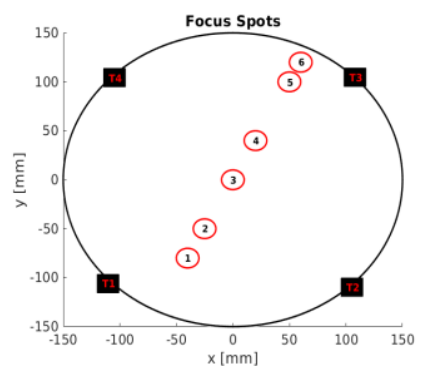

Fig. 3. Locations of target points and transducers in configuration A.

In transducers configuration $\mathrm{B}$, the acoustic field was measured by scanning a xy-plate inside the container with the same hydrophone. The scan was performed twice with different transmitted signals. First, a 20-cycle sine wave was transmitted. Second, TR signal was transmitted to an arbitrary target point (cfr fig. 4). The pressure signals were recorded with a PicoScope (4 channels, 3000 series) and the scan was automated with a custom-made Matlab program. The spatial resolution was $5 \mathrm{~mm}$. The experimental scan results were compared to FEM-simulated predictions.

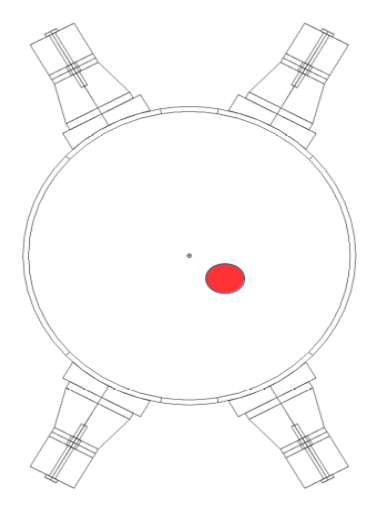

Fig. 4. Location of target with transducer configuration B.

\section{RESULTS}

\section{A. Comparing intensities}

With transducer configuration $\mathrm{A}$, we investigated six different locations (shown in the fig. 3). First, we transmitted TR-signal targeted at the location \#1 and measured pressures with the hydrophone at each of the six locations. The measurement was repeated with other TR signals, targeted to 
locations \#2-6. In each of the cases, the highest intensity was measured at the targeted location. Figure 5 A-C shows normalized intensities at target points \#1, \#3 and \#5.

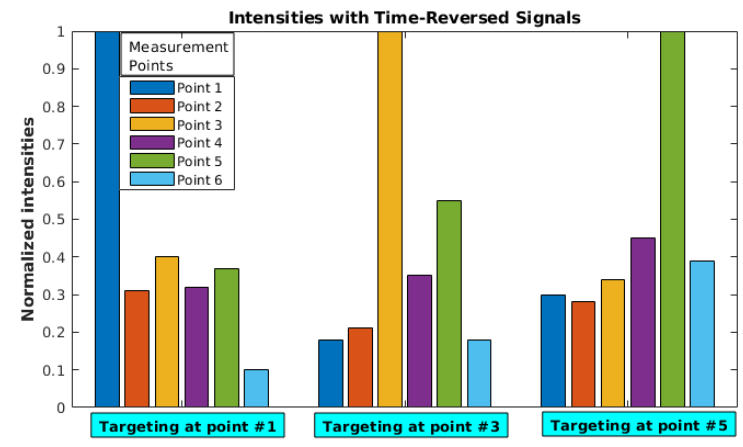

Fig. 5 A. Intensities at six locations, when focusing on target point \#1, \#3, and \#5, respectively. The highest intensity indicates the achieved focal point.

\section{B. Comparing pressure fields}

With the transducer configuration B, pressures were plotted as colormaps with transmitted sine waves (fig. 6) and with transmitted TR signals (fig. 6). Colormaps were compared to the FEM predictions.
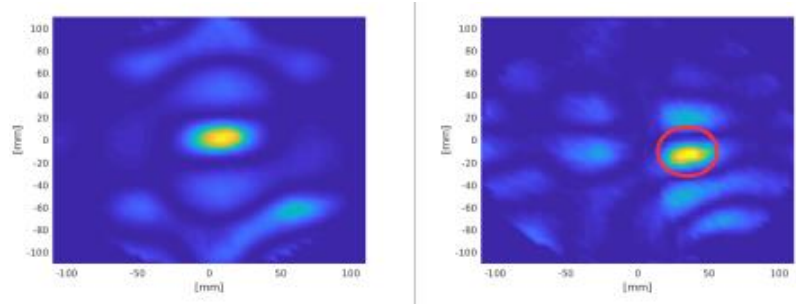

Fig. 6. Comparing experimental pressure fields with the transmitted sine wave (left) and with the TR-signal (right). With symmetrical mounting, transmitted sine wave focuses on the center. With the TR-signal, the target point is circled. Color scale is normalized and proportional to the intensity.

\section{CONCLUSION}

We focused ultrasound with only four Langevin transducers onto predefined target points inside a homogenous media using the TR technique. We did this using simulated forward propagated signals.

\section{REFERENCES}

[1] M. Fink, "Time Reversal of Ultrasonic Fields - Part I: Basic Principles", IEEE Transactions of Ultrasonics, Ferroelectrics and Frequency control, vol. 39, pp. 555-566, September 1992.

[2] J-L. Thomas, F. Wu, M. Fink, "Time Reversal Focusing Applied to Lithotripsy”, Ultrasonic Imaging., vol. 18, pp.106-121, April 1996.

[3] J. Gâteau, L. Marsac, M. Pernot, J-F. Aubry, M. Tanter, M. Fink, "Transcranial ultrasonic therapy based on time reversal of acoustically induced cavitation bubble signature", IEEE Trans Biomed Eng., vol. 57 pp. 134-144, January 2010.

[4] S. Yon, M. Tanter, M. Fink, "Sound focusing in rooms: The time-reversal approach", The Journal of the Acoustical Society of America, vol 113, pp. 1533-1543, January 2002

[5] B. Svedin, M. Beck, J. Hadley, R. Merrill, J. de Bever, B. Bolster Jr., A. Payne, D. Parker, "Focal Point Determination in Magnetic Resonanceguided Focused Ultrasound Using Tracking Coils", Magn Reson Med., vol. 77, pp. 2424-2430, July 2017.

[6] C.A. Speed, "Therapeutic ultrasound in soft tissue lesions", Rheumatology, vol. 40, pp. 1331-1336, December 2001.

[7] R. Apfel, C. Holland, "Gauging the likehood of cavitation from shortpulse, low-duty, cycle diagnostic ultrasound", Ultrasound Med \& Bio, vol. 17, pp. 179-185, 1991 